\title{
SUBCLUSTERS IN THE VIRGO AREA
}

\author{
Z.Y. SHAO \\ Shanghai Astronomical Observatory \\ 80 Nandan Road, Shanghai 200030, China \\ E-mail:zyshao@center.shao.ac.cn
}

By using the data of the CfA redshift catalogue(1995.4), the structure, substructures and some astrophysical properties of the Virgo galaxy cluster are studied in depth (Shao 1996).

At first, a method is developed to study the characteristical scales of substructures in galaxy clusters by counting the peak numbers of wavelet coefficients for consecutive wavelength. It is found that there were three special scales related to typical radius of subclusters in the Virgo area, $135^{\prime}(\sim 600 \mathrm{kpc}), 9^{\prime}(\sim 400 \mathrm{kpc})$ and $60^{\prime}(\sim 270 \mathrm{kpc})$.

Secondly, a universal, self-consistent mathematical model is established and applied to determine distribution parameters of 10 subclusters in the Virgo area. The membership probability(MP) of individual galaxies are caiculated, for example, IMPs of M100 and NGC4571 belonging to the subcluster $\mathrm{A}(\mathrm{M} 87)$ is 0.90 and 0.82 , respectively.

Furthermore, the structure and kinematics of the Virgo area are discussed as follows: (1) Subcluster A is the dominant component of the Virgo. Subcluster B(M49) is some 0.6 magnitudes farther than A, and falling towards it from behind. Subcluster $\mathrm{W}$ is situated in the remote place of the Virgo area. (2) Other small clumps, such as $M_{1}, M_{2}, A_{n}, W^{\prime}$, etc., which mainly consist of late type galaxies, are setting in the halo of subcluster $\mathrm{A}$. There are some evidences which indicate that they are infalling towards the center of A. (3) Comparing with the whole Virgo cluster, subcluster A has a bit more enough dynamical evolution. It has a dense core that consists of early type galaxies. In the core, galaxies are interacting violently, but still far away from virialization. Late type galaxies are infalling from the halo towards the core. (4) The best estimate of the mean radial velocity for subcluster $A$ is $1055 \pm 42 \mathrm{kms}^{-1}$ (heliocentric).

\section{Reference}

Shao, Z.Y., 1996, Ph.D. thesis, Shanghai Astronomical Obs. 